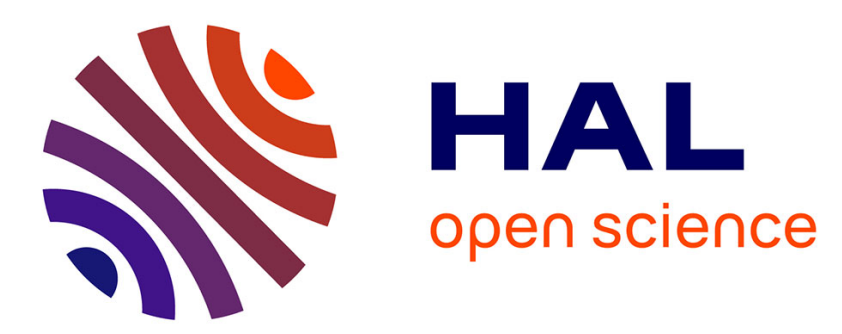

\title{
Comptabilité (publique et d'entreprise) et sociologie, ou l'analyse sociologique des catégorisations sociales
}

Corine Eyraud

\section{To cite this version:}

Corine Eyraud. Comptabilité (publique et d'entreprise) et sociologie, ou l'analyse sociologique des catégorisations sociales. Comptabilité - Contrôle - Audit, 2004, pp.29-45. hal-00154946

\author{
HAL Id: hal-00154946 \\ https://hal.science/hal-00154946
}

Submitted on 15 Jun 2007

HAL is a multi-disciplinary open access archive for the deposit and dissemination of scientific research documents, whether they are published or not. The documents may come from teaching and research institutions in France or abroad, or from public or private research centers.
L'archive ouverte pluridisciplinaire HAL, est destinée au dépôt et à la diffusion de documents scientifiques de niveau recherche, publiés ou non, émanant des établissements d'enseignement et de recherche français ou étrangers, des laboratoires publics ou privés. 


\title{
Comptabilité (publique
} et d'entreprise) et sociologie, ou l'analyse sociologique des catégorisations sociales

\author{
Corine EYRAUD
}

\section{Résumé}

Nous nous intéressons à la comptabilité en tant que système de catégorisations sociales, et mobilisons les travaux sociologiques réalisés sur la construction des groupes sociaux et la catégorisation qui l'accompagne. A travers deux exemples, celui de la réforme comptable des entreprises chinoises et celui de la réforme de la comptabilité budgétaire française, nous tenterons de montrer, d'une part, ce que l'analyse sociologique des catégorisations sociales peut apporter à l'étude de la comptabilité, et, d'autre part, l'intérêt pour le sociologue d'intégrer la question comptable dans son analyse des phénomènes économiques et sociaux.

Mots Clés. - SOCIOlOgIE - CATÉgorisations SOCIALES - COMPTABILITÉ D'ENTREPRISE, COMPTABILITÉ PUBLIQUE - L.O.L.F.

\section{Abstract :}

We look at accounting as a system of social categorizations and have taken into account sociological research done on the construction of social groups and the categorization which goes along with it. Using two examples, that of the accounting reform of Chinese companies and that of the reform of French budgetary accounting, we attempt to show, on the one hand, that the sociological analysis of social categorizations can bring a lot to the study of accounting and, on the other hand, that it is the interest of the sociologist to include the accounting question in his analysis of economic and social phenomena.

KEYWORDS. - SOCIOLOGY - SOCIAL CATEGORIZATIONS - ACCOUNTING - GOVERNMENT ACCOUNTING - L.O.L.F.
Correspondance : Corine EYRAUD

Laboratoire méditerranéen de sociologie (LAMES) -

Département de sociologie,
Université de Provence,

29, avenue Robert Schuman,

13621 Aix-en-Provence cédex 1, France

Email : eyraud@up.univ-aix.fr 


\section{Introduction}

Une fois passés les travaux pionniers de Marx, Sombart et Weber, la sociologie s'est très peu intéressée à la question comptable (Miller, 2000). De manière générale, et ce jusqu'aux années 1980, la sociologie a délaissé, dans ses analyses, les domaines d'activités à forte tendance technique, que ces domaines soient de l'ordre de la technologie, de la science, des statistiques ou de la comptabilité. Deux raisons peuvent, nous semblent-ils, être évoquées. La première tient aux compétences techniques nécessaires à l'analyse sociologique de ces domaines particuliers, compétences dont peu de sociologues étaient pourvus. La deuxième tient à l'acceptation par la discipline sociologique de la séparation entre ce qui est de l'ordre du social et ce qui est de l'ordre du technique (ou de l'économique).

Ce sont les travaux réalisés en sociologie des sciencesi par Latour et Callon, dans les années 1980, qui remettront en cause ce cloisonnement des activités et des domaines de recherche, montrant, entre autres choses, que cette division est socialement construite, qu'elle est un des enjeux des rapports sociaux et certainement pas une donnée : "Les controverses ne deviennent vraiment techniques que lorsque les rapports de force ont été stabilisés et lorsque sont connus les noms des vainqueurs possibles. Par contre dans les moments initiaux, un des principaux enjeux est de délimiter la sphère de la technique et les enchaînements qui lui sont imputables" (Callon, 1981, p. 391). D'après ces auteurs, c'est par la controverse que s'élaborent les faits (scientifiques, technologiques, etc.) ; une fois constitué, le fait devient une boîte noire qui ne donne rien à voir d'elle-même. Pour l'analyser, il convient de le suivre en train de se faire et/ou de " rouvrir les boîtes noires " fermées par les acteurs. Or pour le sociologue, comme pour beaucoup de citoyens, la comptabilité relève à la fois du domaine économique et du domaine technique, et constitue bel et bien une boîte noire, qui plus est hermétique, aux deux sens du terme.

Comme Miller (2000) le soulignait, la mise en perspective sociologique de la comptabilité a, depuis les années 1980, beaucoup plus été le fait des sciences comptables et de gestion que des sociologues. Ceci étant, de nombreux travaux de sociologie, réalisés justement à partir des années 1980, peuvent être mobilisés de manière heuristique. Nous nous intéresserons ici à la comptabilité en tant que système de catégorisations sociales, et mobiliserons essentiellement les travaux réalisés sur la construction des groupes sociaux et la catégorisation sociale qui l'accompagne, en particulier ceux de Boltanski sur les cadres (1982), de Desrosières et Thévenot sur les catégories socio-professionnelles (1988) puis les différents travaux de Desrosières sur les statistiques. C’est à leur présentation et à la mise en évidence des éléments qui peuvent ici nous intéresser que sera consacrée la première partie de cet article. Il s'agira ensuite de montrer comment ces approches peuvent être appliquées à l'étude sociologique de la comptabilité, et ce à travers deux exemples : celui de la réforme comptable des entreprises chinoises mise en place à partir de 1993 (deuxième partie), et celui de la réforme en cours de la comptabilité budgétaire française en particulier concernant les universités (troisième partie). Chacun de ces exemples s'appuie sur des enquêtes de terrain conséquentes alliant analyse documentaire, entretiens et observation ${ }^{2}$.

Comptabilité - ConTrôle - Audit / Numéro spécial - Mai 2003 (p. 169 à 187) 


\section{L'analyse des catégorisations sociales en sociologie}

Notre objectif n'est évidemment pas l'exhaustivité. Il serait en effet possible d'écrire un ouvrage sur cette question, remontant à Durkheim et à ses premiers travaux sur les représentations collectives (1912). Nous commencerons ici avec le travail de Boltanski, Les cadres - La formation d'un groupe social (1982). Au départ, ce sociologue puise des éléments notamment dans la sociologie de Bourdieu, en particulier sa réflexion sur "les luttes de classement ", c'est-à-dire les luttes symboliques autour de la définition des classes sociales, de leurs frontières et de leurs positions respectives. En cela, cette approche s'écarte des problématisations classiques des groupes sociaux qui partent de l'évidence de l'existence du groupe comme une chose bien délimitée et délimitable, enracinée dans l'ordre de l'économie et/ou de la technique. Boltanski s'efforce de s'émanciper de ce substantialisme en reconstituant l'histoire de la catégorie des cadres, " en étudiant les conditions sociales et politiques qui ont rendu possible la formation du groupe et les luttes politiques qui ont eu pour enjeu sa définition et sa délimitation" (p. 53). Le groupe des cadres, dont Boltanski, pourrait-on dire, rouvre la boîte noire, est un groupe qui a réussi, dans le sens où il « est parvenu à assurer sa cohésion, à imposer la croyance dans son existence et à s'objectiver dans des institutions " (p. 54) administratives et statistiques (nomenclature des catégories socio-professionnelles), syndicales, ou de protection sociale (caisse de retraite des cadres, etc.).

De ce travail nous retiendrons quatre idées essentielles. Premièrement, une catégorie se construit par un plus ou moins long processus historique, dans des rapports de force entre acteurs sociaux. Deuxièmement, la séparation même externe/interne, autrement dit la délimitation de la catégorie, est un des enjeux et un des produits de ce processus et de ces luttes. Troisièmement, ce qui est à l'œuvre dans ces luttes de classement, dans l'invention et la délimitation des catégories, dans les définitions concurrentes, c'est une représentation de la société (non pas constituée de deux classes antagonistes, mais, justement en lutte contre une telle conception, une représentation ternaire centrée sur la classe moyenne). Quatrièmement, c'est " le travail d'objectivation (de la catégorie) qui, en enracinant le groupe dans des institutions et des organisations, lui fait prendre conscience de son existence et, par là, se réaliser " (p. 233), autrement dit les catégories peuvent avoir une certaine efficacité sociale, un " effet en retour» sur la réalité.

Ces quatre idées se retrouvent largement dans différents travaux ultérieurs sur les catégorisations sociales, en particulier dans les recherches portant sur les statistiques initiées par Desrosières et Thévenot à propos des catégories socio-professionnelles (1988), puis poursuivies par Desrosières dans différents domaines de la statistique $(1993,2000)$. On trouve ainsi confirmation, à propos de la nomenclature des CSP de manière générale puis des catégories et sous-catégories ${ }^{3}$ qui la constituent, de la validité de ces quatre idées. On ne reprendra donc pas ce travail, si ce n'est pour souligner la proximité d'une telle nomenclature, de cet ensemble de catégories et sous-catégories qui font système et que Desrosières nomme "des nomenclatures emboîtées " (2000, p. 293), avec la " nomenclature gigogne » qu'est le plan comptable (Colasse, 2003, p. 166). Finalement, ce travail sur les CSP montre, un peu plus précisément que celui sur les cadres et à propos d'une nomenclature et non plus seulement d'une catégorie isolée, qu'une telle nomenclature est de l'histoire accumulée (histoire économique, sociale et politique), et qu'en elle est inscrite une certaine vision de la société. Desrosières pourra par la suite énoncer " qu'il est possible de regarder en même temps des philosophies sociales ou politiques et des outillages en apparence technique, en les considérant comme un tout» (2000, p. 84).

Comptabilité - ConTrôle - Audit / Numéro spécial - Mai 2003 (p. 169 à 187) 
Corine EYRAUD

La question de l'efficacité sociale des catégories, dernière idée retenue, a été travaillée par de nombreux sociologues. Bourdieu, déjà, insistait sur le fait que les catégories ne sont pas seulement descriptives mais également prescriptives (voir par exemple 1993). Les ethnométhodologues ${ }^{4}$ ont poursuivi cette réflexion et, rompant plus radicalement avec la doctrine représentationnaliste et une certaine sociologie de la connaissance, ils ont mis en évidence le caractère opératoire des catégories, ne les considérant pas comme des représentations mais comme des schèmes, "c'est-à-dire des règles ou des méthodes (pour une part incorporées) gouvernant les activités ou les opérations de construction de l'objectivité " (Quéré, 1994, p. 32), " des formules d'opérations possibles ou des méthodes pour des activités pratiques (de détermination, de prédication, d'individualisation, de classification, de configuration, d'organisation de cours d'action, etc.) » (id., p. 37).

La question se pose un peu différemment quand il s'agit de nouvelles catégories, de mise en place de nouveaux systèmes de catégorisation, comme c'est le cas dans les deux exemples que nous allons développer. L'efficacité de ces nouveaux systèmes, en terme de changement des pratiques et des représentations, est alors soumise à un certain nombre de conditions. Pour réfléchir à ces conditions et en conservant l'idée que les systèmes de catégorisation sont des matérialisations ou des inscriptions de théories, de philosophies sociales et économiques, plusieurs écoles de la sociologie peuvent être mises à contribution. Nous pouvons ainsi reprendre les analyses de Bourdieu sur les conditions sociales des " effets de théorie " (1982, en particulier pp. 156-161), c'est-à-dire des effets que peut avoir une théorie philosophique et/ou sociologique sur le monde social : la réalité de ces effets est subordonnée au fait que des agents s'approprient des éléments de cette théorie et que celle-ci puisse s'appuyer sur des institutions. Bourdieu a ainsi réfléchi aux conditions pour qu'une théorie s'impose ; la sociologie des sciences a analysé la genèse historique de nombreux faits scientifiques (Callon, 1989 ; Latour, 1984, 1989) mettant au jour les conditions pour qu'un fait réussisse, c'est-à-dire devienne un fait (un énoncé qui n'est plus contesté par les pairs-concurrents) et se solidifie comme tel. Elle a ainsi montré que la construction d'un fait scientifique ne renvoie pas seulement à un travail intellectuel et discursif, mais mobilise tout un ensemble d'autres pratiques ainsi que des techniques et des objets qui sont des matérialisations de débats antérieurs. Finalement, la réussite ou la réalité d'un fait dépend de l'extension et de la robustesse du réseau plus large des objets, acteurs et institutions dans lequel il s'inscrit ${ }^{5}$. On comprend alors que la mise en place de nouvelles conventions, de nouvelles nomenclatures soit très coûteuse en termes de temps, de mobilisations, d'inscriptions.

Ce rapide panorama sur l'approche sociologique des catégorisations sociales serait incomplet si nous n'abordions pas la question de la réalisation des catégories par les acteurs sociaux, ou autrement dit les pratiques de classement. Dans le domaine des statistiques, on appelle ce moment «l'opération de codage, qui affecte des cas singuliers à des classes " (Desrosières, 1993, p. 16), et constitue donc "le passage de la singularité à la généralité " (id., p. 290). Le codage est ainsi "l'acte fondamental de reconnaissance et de désignation (" ceci est un... ») qui mobilise le savoir antérieur, le fait revivre à nouveau en réactivant une catégorie, comme un sentier ne survit que s'il est régulièrement parcouru " (id., p. 339). Desrosières et Thévenot (1988, pp. 54-66) ont analysé les pratiques de classement de "professionnels " (employés à la codification) et de "profanes » en imaginant un exercice expérimental : les participants devaient construire une nomenclature socioprofessionnelle en répartissant en plusieurs tas un paquet de fiches contenant des informations du type de celles figurant sur un questionnaire statistique, puis nommer chacun des tas constitué. Les chercheurs ont mis en évidence que ces pratiques de classement procèdent par rapprochement et identification aux " bons exemples ",

Comptabilité - Contrôle - Audit / Numéro spécial - Mai 2003 (p. 169 à 187) 
beaucoup plus que par une définition de critères ; or ces bons exemples, ces images typiques des catégories (« le cadre », etc.) doivent beaucoup au travail de représentation politique des groupes sociaux. Ces conclusions sont également valables pour les " professionnels " car de nombreux cas particuliers les obligent à s'écarter des consignes strictes. L'ethnométhodologie a, bien évidemment, beaucoup travaillé cette question, puisqu'elle se veut "une compréhension détaillée des méthodes des membres (d'une collectivité) pour produire et reconnaitre leurs objets sociaux, événements, activités " (Coulon, 1987, p. 86). L'idée de base est que le sens des catégories est un sens situé, dépendant du contexte, et que l'ethnométhodologie doit décrire cet accomplissement des catégories. Cela requiert une description fine des activités pratiques. Nous ne nous intéresserons pas, dans la suite de cet article, à la question de la réalisation des catégories, il faudrait d'autres matériaux d'enquête que ceux dont nous disposons pour l'instant : observation de pratiques de comptables (et non pas des entretiens, même approfondis, qui produiraient une rationalisation a posteriori de leurs codages) et/ou réalisation de petits exercices comme ceux réalisés pour les nomenclatures socioprofessionnelles.

Finalement, nous définirons la comptabilité comme une "nomenclature gigogne " (la comptabilité normalisée) actualisée, réalisée par des pratiques de codage ou d'enregistrement (la comptabilité pratiquée) ; et c'est à la première de ces dimensions que nous allons nous intéresser.

\section{La réforme comptable chinoise}

\subsection{De la définition de l'entité et de quelques catégories comptables}

Comparer deux systèmes comptables si différents que celui de l'économie planifiéevi (élaboré à partir du modèle soviétique) et celui mis en place par la réforme de 1993 permet de mettre en évidence le caractère construit historiquement de la comptabilité, et éclaire un certain nombre de débats et propositions récents, même si largement minoritaires.

Pendant la période de l'économie planifiée, les entreprises d'État chinoises émargent au budget de l'État ; c'est ce dernier qui finance les besoins de trésorerie et les investissements, absorbe les profits et éponge les pertes. Les entreprises sont finalement un simple maillon de l'économie nationale et du budget de l'État, leur comptabilité relève du domaine de la comptabilité publique. Le jeu de documents comptables comprend la "balance de fonds " ${ }^{7}$, le " tableau des profits ou des bénéfices " et des "tableaux de coûts ». La balance de fonds se compose de deux parties équivalentes (les fonds alloués et les fonds utilisés), chacune divisée en trois catégories : les fonds fixes, les fonds circulants et les fonds spéciaux. La gestion financière des entreprises doit obéir au principe "d'utilisation des crédits conformément à leur affectation ».

Les définitions de nombreuses catégories comptables sont directement liées aux concepts de l'économie politique marxiste. Les fonds fixes, par exemple, comprennent les bâtiments professionnels, équipements et machines mais aussi tout " ce qui rend possible pour les travailleurs la réalisation de leur travail sur une longue période " : logements, crèches et écoles pour les enfants, dispensaire, etc. Il s'agit clairement d'une tout autre idée et d'une tout autre forme d'entreprise que celle que nous connaissons aujourd'hui en Occident : c'est l'entreprise qui assume les coûts de production de la force

Comptabilité - ConTrôle - Audit / Numéro spécial - Mai 2003 (p. 169 à 187) 
Corine EYRAUD

de travail, ces coûts sont intégrés aux coûts de production stricto sensu. Le profit, d'autre part, est, dans la théorie marxiste, la valeur ajoutée par les travailleurs. Les impôts sont donc considérés comme du profit transféré à l'État, les frais financiers comme du profit transféré aux banquesviii. Ainsi, dans le système comptable de l'économie planifiée chinoise, le bénéfice ou profit comprenait les impôts, les frais financiers, le profit distribué aux employés par le fonds de bien-être et par les primes, le profit remis à l'État, et celui conservé par l'entreprise. Les impôts, les frais financiers, les primes des employés (mais non pas les salaires) n'étaient donc pas considérés comme des charges mais comme des répartitions du bénéfice.

Les règlements instaurant la réforme comptable chinoiseix (1993) définissent les nouveaux concepts utilisés en comptabilité : actif, passif, capitaux propres, produit, charge, profit et perte. Ils définissent également les nouveaux documents comptables, qui seront désormais un bilan, un compte de résultat, un tableau de financement, un tableau de répartition du bénéfice et une annexe. Le principe d'utilisation des crédits conformément à leur affectation est supprimé. Ce nouveau système comptable, avec la notion de bilan, de capitaux propres se fonde sur un certain modèle de l'entreprise : une entreprise est une entité à part entière, elle n'est plus un simple élément du système économique national et du budget de l'État. La comptabilité des entreprises chinoises devient ainsi une comptabilité privée, une comptabilité d'entreprise au sens capitaliste du termex : elle vise à évaluer un patrimoine et à calculer un résultat. Si l'on ajoute à cela le fait que les immobilisations non professionnelles (logements des employés, crèches, etc.) ne doivent plus figurer dans le bilan des entreprises, on voit se dessiner plus précisément le modèle de l'entreprise qui fonde cette réforme comptable : une entité essentiellement économique qui ne doit plus assumer ni les coûts de production de la force de travail ni la gestion de cette production, mais qui externalise et ces coûts et cette gestion en vendant les logements, en confiant les crèches et écoles à la société globale, etc.

La définition de ces internalités et externalités est donc à la fois inscrite dans, et produite par, les documents et catégories comptables. Or on se rend compte aujourd'hui que les choix liés à l'activité de production ont également des coûts en terme par exemple de chômage et de pollution. Ces coûts ne sont pas comptabilisés dans les coûts de revient des produits de l'entreprise ni dans ses charges indirectes, mais externalisés, même si l'entreprise en assume une partie par le versement de cotisations et le paiement d'impôts. Or on peut penser que ce système d'externalisation a des effets négatifs non seulement sur les salariés et la société globale, mais également sur l'entreprise elle-même (Salais et al., 1986).

Dans cette optique, un groupe de chercheurs français a tenté récemment, à partir d'une initiative du Centre des jeunes dirigeants de l'économie sociale (CJDES) et en s'inspirant des travaux des sociologues Boltanski et Thévenot, d'internaliser ces externalités au sein d'un bilan particulier qu'ils ont appelé " bilan sociétal " (Capron, Leseul, 1997). Dans une autre direction, le Centre des Jeunes Dirigeants a proposé d'inverser l'équation de base de l'entreprise capitaliste, en considérant que la rémunération du capital devrait être comptabilisée comme une charge, et que la différence entre les produits et les charges - le bénéfice - devrait profiter uniquement aux salariés : "c'est un moyen d'attribuer les résultats aux collaborateurs de l'entreprise qui en sont la finalité, et finalement de remettre la finance au service de l'économie » (CJD, 1996, p. 106) ${ }^{11}$. Cela dit, cette idée n'est pas neuve, elle fut même mise en pratique sous différentes formes par des patrons socialistes et plus précisément fouriériste au $19^{\mathrm{e}}$ siècle (par exemple par Godin dans son Familistère de Guise ${ }^{12}$ ), par des patrons

Comptabilité - Contrôle - Audit / Numéro spécial - Mai 2003 (p. 169 à 187) 
catholiques sociaux au 20 $0^{\mathrm{e}}$ siècle (par exemple Dubois dans les Forges et Aciéries de Bonpertuis) ${ }^{13}$, et d'une manière un peu différente par les entreprises autogérées de la Yougoslavie de Titoxiv.

Colette et Richard font « l'hypothèse que l'agent économique qui a le pouvoir (qui domine) dans un système économique donné, façonne le système comptable (et donc le compte de résultat) de façon à faire apparaître en priorité "son » résultat, calculé selon sa propre vision de la réalité économique " (2002, p. 13). Ainsi, tout concept de résultat se fonde sur une conception politique de l'économie, sur une vision de l'économie, de ses objectifs, de ce qu'est la richesse créée et de ce que sont ses destinataires ; la vision qui domine à un moment donné dans une société donnée fonde donc le concept de résultat utilisé. Cette vision s'est construite dans un processus historique fait d'alliances, de constitution d'acteurs et de groupes d'acteurs, de rapports de force, de compromis ; elle s'est progressivement solidifiée, institutionnalisée dans un certain nombre de dispositifs, le dispositif comptable étant l'un d'entre eux.

\subsection{De l'efficacité de la réforme}

Nous pouvons tenter d'évaluer cette efficacité sociale en analysant deux de ses possibles dimensions : l'action de cette réforme premièrement sur les représentations - et ce à travers l'émergence de nouvelles catégories économico-comptables chez les acteurs eux-mêmes -, deuxièmement sur les pratiques - à travers l'utilisation des bilans comptables dans les décisions gouvernementales de mises en faillite ${ }^{15}$.

Concernant la première de ces dimensions, nous pouvons prendre comme exemple les notions de prêt, d'investissement, de subvention, de dettes et de créances ; toutes sont des nouvelles catégories définies par la réforme comptable. Trois ans après la mise en place de cette réforme (enquête de 1996), elles sont encore largement confondues dans les discours des directeurs d'entreprise et du personnel bancaire : tous considèrent les comptes clients comme des prêts, et font peu de différence entre une subvention, un prêt bancaire et un investissement de l'État. L'idée que tout cet argent provient de la même poche, de la "grande marmite commune " est encore très prégnante. C'est ce qu'exprime un directeur d'entreprise : « Nous devons de l'argent à des entreprises d'État qui nous doivent de l'argent, nous devons de l'argent aux banques d'État, nous devons de l'argent à l'État, nous sommes une entreprise d'État. Pour moi, c'est absurde, c'est le Parti communiste qui doit au Parti communiste ». Au début des années 2000, la dissociation de ces catégories est largement plus prononcée : le terme de "prêts ", par exemple, n'est plus utilisé pour parler des créances clients mais uniquement pour parler de prêts bancaires. Cette dissociation n'est toutefois pas aboutie ; un directeur d'entreprise nous signale : "Une banque est venue investir dans notre entreprise ", et répond à notre demande d'explicitation (" investir ou prêter de l'argent ? ") : "C'est pareil, en fait elle nous a donné un prêt ».

Cela dit, de manière générale, la réforme comptable participe au développement de l'esprit de calcul, ce qui n'a rien d'étonnant : "Avec cette nouvelle comptabilité, les profits apparaissent clairement, je vois comment les augmenter : il faut compter. J'ai besoin de tel matériau, à tel endroit il est à 9600 yuan la tonne, à tel autre à 10000 yuan, le premier représente une économie, il faut que nous ayons un esprit de calcul. De la même manière, il faut diminuer les gaspillages en matières premières et en produits finis ». Et participe également au développement du " sentiment d'entité ": "Cette réforme renforce la notion de gestion interne, de capitaux propres, elle augmente la conscience d'autoexploitation» (citations d'entretiens de directeurs d'entreprise).

Comptabilité - ConTrôle - Audit / Numéro spécial - Mai 2003 (p. 169 à 187) 
Corine EYRAUD

Concernant la deuxième dimension, nous insisterons ici seulement sur un de ses aspects : celui visà-vis duquel la réforme comptable est d'une efficacité féroce c'est, depuis 1997, dans la mise en faillite et les plans de licenciement.

Le président chinois de l'époque, Jiang Zemin, dans son discours au $\mathrm{XV}^{\mathrm{e}}$ congrès du Parti en septembre 1997, prend une position tranchée sur la question des sureffectifs des entreprises d'État, et déclare qu'il faut " choisir une réduction du personnel pour une augmentation des résultats " ${ }^{16}$. Le chef du gouvernement de l'époque, Zhu Rongji, annonce, quant à lui, que le problème des pertes des entreprises d'État, devenu visible avec l'adoption de la nouvelle comptabilitée ${ }^{17}$, doit être réglé dans les trois ans. Une pression énorme sur les gouvernements locaux s'ensuit ; une grande campagne d'analyse des bilans des entreprises d'État est lancée, de repérage de celles connaissant des pertes importantes et chroniques, d'autorisations à partir de ce repérage de plans de licenciement, de préretraites et de mises en faillite. Le bilan, quoique difficile à chiffrer précisément, est considérable. Sur l'ensemble de la période 1994-2000, on estime qu'aurait été "descendus de poste " de 36 à 55 millions de personnes, soit de un tiers à la moitié des employés d'entreprises d'État répertoriés en 1994, ou de 20 à $30 \%$ de l'emploi urbain de cette même année. Ces estimations ne rendent pas complètement compte de l'ampleur du phénomène, en particulier elles laissent de côté la pratique de la mise en retraite anticipée : parfois dès 40 ans pour les femmes, 50 ans pour les hommes, et avec un salaire très réduit.

\subsection{Du mode de régulation de la société qui l'accompagne}

Nous n'insisterons pas sur les modifications profondes et évidentes des relations État/entreprises entre l'ancien système comptable (utilisation des crédits conformément à leur affectation et contrôle de cette utilisation) et le nouveau. Il nous paraît plus intéressant de porter notre regard à un niveau plus macro, celui du type de normalisation à l'œuvre, avant et après la réforme.

A partir du début des années 1950, il existe en Chine plus d'une cinquantaine de "systèmes comptables » et de "systèmes financiers ». Les premiers comprennent un plan comptable et un modèle type de chaque document comptable, les seconds sont des textes très détaillés et très directifs expliquant le contenu de chaque item comptable, sa méthode de comptabilisation, etc. Ces systèmes comptables et financiers different selon le type d'entreprise (entreprises collectives ou entreprises d'État) et selon le secteur de production, car chaque ministère industriel les élabore pour son propre secteur à partir des directives du ministère des Finances et du bureau national des Statistiques ${ }^{18}$. Ces systèmes comptable et financier ont valeur de règlements ; la division comptabilité du ministère industriel, mettons du ministère de la Sidérurgie, les transmet aux services comptabilité des bureaux provinciaux de la sidérurgie qui les transmettent aux services comptabilité des bureaux municipaux de la sidérurgie, qui les transmettent finalement aux sections comptabilité des entreprises qui leur sont subordonnées. L'information comptable en provenance des entreprises effectue essentiellement le chemin inverse.

On retrouve dans ce mode de gestion de l'information et des systèmes comptables et financiers, les principaux éléments du système chinois de régulation de l'économie et, plus largement, de régulation/gestion de la société. Il s'agit de ce que nous avons appelé « le système de gestion échelon par échelon " (Eyraud, 1999) : il existe très peu de lois, tout est encadré par des règlements transmis en suivant les lignes administratives qui relient hiérarchiquement les organisations chinoises, et pouvant

Comptabilité - Contrôle - Audit / Numéro spécial - Mai 2003 (p. 169 à 187) 
être modifiés (en raison de conditions sectorielles ou locales particulières) par un ministère ou par les échelons provinciaux et municipaux. Il s'agit finalement d'un type de gestion très particulariste.

A partir de la réforme comptable, les nouveaux règlements sont applicables à toutes les entreprises installées sur le sol chinois, quels que soient leur statut et leur secteur de production. Les systèmes comptables et financiers tendent à disparaître, et devront être remplacés par un seul plan comptable, lui aussi applicable à tous. En cela, cette réforme s'inscrit dans un changement fondamental de mode de gestion de la société : le passage d'une micro-gestion particulariste de l'échelon inférieur par l'échelon supérieur à une régulation universaliste par la loi et par des organismes spécialisés dans une activité donnée (ici le ministère des Finances et les organismes professionnels comptables). Ce changement est en cours dans la société chinoise ; dans de nombreux domaines d'activités, le gouvernement fait un effort important de codification et crée des organismes spécialisés en charge d'élaborer ce droit, de protéger ces droits, de diffuser et conserver l'information.

\section{Réformes de la comptabilité universitaire et de la comptabilité budgétaire françaises}

\subsection{De la définition de l'entité et du mode de régulation qui l'accompagne}

Jusqu’à la fin des années 1980, le budget des universités est composé de différentes lignes budgétaires qui reprennent les catégories et classifications budgétaires de l'État (en chapitres, articles et paragraphes). Ces lignes budgétaires sont gérées par différentes directions ministérielles et allouées sur critères, on trouve par exemple les crédits de fonctionnement matériel et pédagogique (chapitre 3611, matériel), les crédits de vacation et cours complémentaires (chapitre 36-11, personnel), les subventions d'équipement ou renouvellement de matériel (chapitre 66-70), les subventions au titre de la bibliothèque, de la médecine préventive, du CIO, de chaque IUT et école d'ingénieurs, etc. ; le principe régissant l'utilisation des crédits est celui « de l'utilisation des crédits conformément à leur affectation ". Cette " période " est assimilée par la plupart des analystes (voir par exemple Musselin, 2001) à une forte centralisation : les décisions sont prises de façon détaillée au niveau central, ce dernier définit les actes, leurs coûts, et attribue les financements correspondants. Cette forte centralisation serait basée sur des principes d'uniformité et d'égalité (sur le territoire pour la même discipline), principes inscrits dans des normes telles que San Remo, et des procédures telles que les habilitations de diplômes.

A la fin des années 1980, une politique contractuelle est mise en place ; le budget des universités est alors composé d'une Dotation Globale de Fonctionnement (DGF) et de dotations sur projets contractés dans le contrat quadriennal signé avec le ministère. Cette réforme a deux objectifs principaux : premièrement, permettre la prise en compte de la diversité des contextes locaux ; deuxièmement, amener les universités à devenir le lieu de définition de politiques et donc d'arbitrages. Mais rapidement on se rend compte que si la dimension contractuelle prend de l'importance, la proportion

Comptabilité - Contrôle - Audit / Numéro spécial - Mai 2003 (p. 169 à 187) 
des crédits fléchés par rapport aux crédits à utilisation libre par l'université (DGF) s'accroît, ce qui est l'effet inverse des objectifs de la réforme.

Une troisième période pourrait commencer dans les temps à venir : le budget des universités pourrait devenir, comme prévu dans le projet de loi sur l'autonomie des universités, un budget global. Pour l'instant, entrent dans le budget de l'université les dépenses de fonctionnement matériel, pédagogique et scientifique - notamment la rémunération des personnels contractuels, les heures complémentaires d'enseignement, les activités de bibliothèque et musée -, et les dépenses d'investissement incluant la maintenance lourde et la mise en sécurité des locaux puisque les universités assument les charges du propriétaire ; mais n'y entrent pas le traitement des fonctionnaires et agents de l'État, les bourses, les investissements immobiliers du contrat de Plan, les comptes des IUFM, des CROUS, etc.

Un certain nombre de voix de professionnels, parlementaires, experts s'élèvent contre cette situation depuis quelques années; ces critiques sont de deux ordres et se trouvent bien résumées dans les rapports de la Conférence des Présidents des Universités. Premièrement « les budgets universitaires actuels n'intègrent qu'une partie modeste des recettes et dépenses liées à l'activité universitaire et les universités (contrairement aux centres hospitaliers) sont rarement perçus comme des puissances économiques par leur environnement local et régional (...). L'objet de la globalisation du budget sera donc le rapprochement du périmètre budgétaire avec le périmètre des moyens mis en œuvre pour l'accomplissement de l'ensemble des missions de l'université " (CPU, 2002, p. 4). Le budget global pourrait donc comprendre tout ce qui n’y entrait pas jusqu'à présent ${ }^{19}$. Deuxièmement, « l'autonomie des universités apparaît comme une condition nécessaire à l'adaptation intelligente de ces établissements aux évolutions profondes qu'ils doivent subir ainsi qu'aux spécificités des contextes dans lesquels ils se développent. (...) Elles doivent bénéficier de conditions de fonctionnement à la fois plus simples et plus souples. (...) Le budget global leur permettrait de disposer de moyens plus larges pour mener une politique propre. » (CPU, 2001, pp. 62-63).

L'État pourrait d'autre part transférer aux établissements qui en font la demande la pleine propriété des biens immobiliers qui sont mis à leur disposition ; les établissements pourraient donc être amenés à établir un bilan, d'autant que le nouveau logiciel comptable des universités, Jefyco, le permet. L'université ne serait ainsi plus un maillon, une " fin de lignes » en provenance du national, mais une entité intégrée et contenant toutes les activités liées à ses missions. On peut noter, sur ce plan-là, une forte ressemblance entre cette évolution et celle connue par les entreprises chinoises.

\subsection{La L.O.L.F. et le " nouvel esprit du management public »}

Cette évolution s'intègre dans un champ beaucoup plus large : celui de la réforme de la comptabilité budgétaire lancée par la Loi organique relative aux lois de finances (L.O.L.F.) adoptée le $1^{\mathrm{er}}$ août 2001 , et qui devrait entrer progressivement en vigueur pour être totalement appliquée en 2006. Jusqu'à son application, le budget de l'État (Ordonnance organique du 2 janvier 1959 relative aux lois de finances) est divisé par ministères / titres / parties / chapitres (compte élémentaire pour la présentation et l'ouverture des crédits votés par le Parlement, environ 800 aujourd'hui) / articles / paragraphes. Avec la mise en place de la LOLF, le budget de l'État sera divisé en : missions (ensemble de programmes concourant à une politique publique définie, par exemple la lutte contre la toxicomanie ; probablement au nombre d'une trentaine) / programmes (ensemble cohérent d'actions relevant d'un même ministère, maximum 100 à 150) / titres (au nombre de 6 : Dépenses de personnel, de fonc-

Comptabilité - ConTrôle - Audit / Numéro spécial - Mai 2003 (p. 169 à 187) 
tionnement, d'investissement, d'intervention, d'opérations financières, charges de la dette de l'État) / catégories (2 à 5 par titre)

La présentation des crédits par titres est indicative, au sein de chaque programme la fongibilité des crédits est acquise, il n'y aura ainsi plus de cloisonnement entre investissement et fonctionnement. La seule limite concerne les dépenses de personnel, il y a là un plafond et une fongibilité asymétrique : il est possible d'employer moins de personnel que le plafond d'emploi prévu par la loi et de les rémunérer plus, ou de redéployer des crédits de masse salariale vers d'autres crédits de fonctionnement ou d'investissement, mais la réciproque est impossible. Apparaissent ici la question de la limitation, voire de la diminution, de l'emploi public, la question des déficits publics, des critères de convergence de Maastricht, etc. Ceci dit, pour le responsable du programme, c'est-à-dire le ministre concerné, puis pour les responsables d'entités sous sa tutelle, cela signifie un gain évident en matière de capacités de décision. Chaque programme fera également l'objet d'un " projet annuel de performance " puis d'un « rapport annuel de performance ».

D'un point de vue comptable, on peut noter trois innovations importantes. Rappelons que jusqu’à présent la comptabilité publique française est une comptabilité de caisse $\left(\right.$ modifiée ${ }^{20}$ ) ou comptabilité des recettes et des dépenses budgétaires ; cette comptabilité de caisse changera peu mais sera accompagnée d'une comptabilité d'exercice et d'une comptabilité de gestion.

Une comptabilité d'exercice ou comptabilité générale sera donc élaborée d'ici le $1^{\text {er }}$ janvier 2005. Il s'agit d'établir une comptabilité en droits constatés, se rapprochant de la comptabilité d'entreprise : " les règles applicables à la comptabilité générale de l'État ne se distinguent de celles applicables aux entreprises qu'en raison des spécificités de son action " (art. 30 de la LOLF), "le droit comptable de l'État est appelé à rejoindre le droit comptable général en se distinguant fortement de la comptabilité budgétaire. Le constat est que si le système actuel de caisse répond bien à l'objectif de respect et de compte-rendu de l'autorisation parlementaire, il ne permet ni d'améliorer la gestion publique, ni de donner une image fidèle et sincère de la situation financière et patrimoniale de l'État. (...) Doté d'un compte de charge par service, voire par programme, chaque gestionnaire sera en mesure de connaître ses propres coûts, donc de les maîtriser et d'améliorer sa performance " (Guillaume et al., 2002, p. 171). De manière générale, il y a une certaine transposition des règles de la comptabilité générale à l'État. Ainsi les principes de régularité, sincérité et fidélité avancés par la LOLF sont ceux qui régissent le plan comptable général applicable aux entreprises; et il est prévu que le plan comptable public en cours de révision se rapproche du plan comptable général.

Afin de mieux connaître les coûts, une comptabilité de gestion ou comptabilité analytique doit également être élaborée, le contrôle de gestion ${ }^{21}$ et l'évaluation développés. La plupart des ouvrages et articles portant sur l'autonomie des universités ou sur la LOLF oppose deux styles d'intervention ou de pilotage de l'État : l'un réglementaire basé sur un contrôle a priori qui est essentiellement un contrôle de régularité consistant à vérifier l'imputation d'une dépense dans la nomenclature budgétaire chapitre-article, l'autre régulateur basé sur une évaluation a posteriori qui serait conséquent avec une plus grande autonomie laissée au gestionnaire et la globalisation et fongibilité des crédits. Tous les ouvrages et discours favorables $^{22}$ à la LOLF considèrent qu'elle est un préalable à, et un élément intrinsèque de, la "modernisation de l'État " : elle fait partie d'un système de gestion de la performance, « système qui a pour objectif d'améliorer l'efficacité de l'action publique par une plus grande autonomie de gestion et son corollaire : une plus grande responsabilité du gestionnaire (...) et de substituer une logique de résultats ou de performance fondée sur le triptyque « objectifs - résultats -

Comptabilité - Contrôle - Audit / Numéro spécial - Mai 2003 (p. 169 à 187) 
contrôle » à l'ancienne logique de strict empilement des moyens » (Guillaume et al., 2002, pp. 13-14). Ce système de gestion de la performance est d'autre part quasi systématiquement associé à l'objectif de transparence de l'action publique.

On peut penser que l'on a affaire au développement d'un nouvel esprit du management public ${ }^{23}$. Cet esprit s'incarne dans des dispositifs, dont un central : la LOLF et les nouveaux dispositifs comptables qui en découlent. On peut d'autant plus parler de "nouvel esprit » que l'on trouve une grande homogénéité de discours au sein des parlementaires, des hauts fonctionnaires, des équipes dirigeantes d'université, voire même des chercheurs en sciences sociales (voir par exemple CPU, 2001, ce colloque sur l'autonomie des universités regroupait ces différentes catégories d'acteurs). Au premier abord, ce nouvel esprit du management public semble très proche du " deuxième esprit du capitalisme " analysé par Boltanski et Chiapello (1999, pp. 103-111), en particulier dans les solutions avancées en matière de management qui sont : la décentralisation, la méritocratie, la direction par objectifs. Et il est intéressant de voir combien ce nouvel esprit semble motiver, mobiliser les équipes dirigeantes universitaires, comme cela a pu motiver et remobiliser les cadres dans les années 1960.

Cet esprit du management public est finalement « dans une relation dialectique avec des dispositifs" (Boltanski, Chiapello, p. 107) : en même temps il les fonde, il les rend possibles, et en même temps ce sont eux qui peuvent permettre sa matérialisation. Ainsi l'élaboration et l'application de la LOLF constitue un champ d'investigation particulièrement intéressant pour le sociologue : de ce nouvel esprit du management public et des acteurs qui le portent et lui donnent forme (Forest, 2003), à la mise en place concrète de ces dispositifs et à leur traduction et efficacité au niveau d'administrations particulières que sont par exemple les universités.

\section{Comptabilité, analyse des catégorisations sociales et sociologie}

Les deux exemples développés ci-dessus ont le mérite de transcender le cloisonnement fréquent entre comptabilité d'entreprise, relevant des sciences comptables et de gestion, et comptabilité publique, relevant de l'administration ou du management publics; et montrent ainsi que les questions des rapports sociaux et des enjeux sociaux à l'œuvre se posent, dans ces deux domaines, de la même manière. Nous pouvons maintenant reprendre les quatre idées que nous avions retenues de l'analyse sociologique des catégorisations sociales, et montrer qu'elles sont tout à fait pertinentes en ce qui concerne la comptabilité.

Premièrement, la comptabilité est le produit de l'histoire. Nous n'insisterons pas tant cela semble évident ; dans nos exemples elle est le produit de réformes. Deuxièmement, la délimitation de l'entité (entreprise ou administrative) est produite par ce processus. La comptabilité véhicule donc une représentation de ce qu'est l'entreprise ou l'entité administrative, de ce qui lui est interne (ses objectifs) et de ce qui lui est externe ; cette représentation est inscrite dans les documents et catégories comptables. Au-delà " des principes de construction du réel » (principes de l'entité, de monétarisation, etc.) dont les effets ont été bien étudiés (Amblard, 2002 ; Colasse, 2003, pp. 52-67 ; Colette et Richard, 2002, pp. 40-66), ce sont les délimitations de catégories (actif, passif, capitaux propres, etc.) et de sous-caté-

Comptabilité - ConTrôle - Audit / Numéro spécial - Mai 2003 (p. 169 à 187) 
gories (immobilisations) qui construisent cette délimitation de l'entité. Un changement de représentation s'accompagne nécessairement d'une transformation, plus ou moins profonde, de ces documents et catégories.

Troisièmement, chaque type de système comptable se fonde sur une certaine conception - non pas, comme dans les statistiques, de la société et de sa structuration - mais de l'économie et de ses objectifs et/ou une certaine conception des services publics, de l'administration et de ses objectifs. Ces visions sont portées par des groupes sociaux, par des acteurs sociaux ; il existe des visions concurrentes se basant chacune sur une délimitation différente de l'entité.

Quatrièmement, un système comptable peut avoir une certaine efficacité sociale, un effet en retour sur la réalité sociale. C’est le point le plus difficile à étudier car il faudrait pouvoir « isoler l'effet comptable "; on peut toutefois souligner que l'effet d'ensemble est possible justement quand l'élément comptable ne reste pas isolé, mais trouve des échos, des alliés, des relais et forment corps et système avec eux.

Mais une autre idée a émergé de chacun de nos deux exemples : un système comptable s'intègre dans, et porte en lui, un certain mode de régulation du social, un certain mode de régulation des entités concernées (entreprises ou administrations), un certain mode de relation entre l'entité, telle qu' elle a été définie par les processus sociaux évoqués plus haut, et l'État.

Le détour par l'analyse des catégorisations sociales montre ainsi son intérêt heuristique, et montre que les approches et techniques de la sociologie peuvent être utiles aux sciences comptables et de gestion $^{24}$. Mais si la sociologie peut être utile au comptable, en quoi la comptabilité peut-elle être utile au sociologue? Nous avons vu qu' " il est possible de regarder en même temps des philosophies sociales ou politiques et des outillages en apparence technique, en les considérant comme un tout " (Desrosières, 2000, p. 84). Partir des outillages ou des dispositifs nous semble une voie intéressante et productive pour le sociologue, parce qu'une fois la difficulté technique dépassée, la lecture des processus à l'œuvre est plus facile et porte moins à discussions : ces processus sont objectivés dans ces changements de dispositifs et ils deviennent finalement relativement évidents. Ceci dit, en matière de gestion publique comme en matière de gestion d'entreprises privées, les dispositifs sont souvent mis en place pensant qu'ils amèneront avec eux la mise en place de la philosophie sociale ou politique qui les sous-tendent, or ils n'en sont qu'un élément qui peut être traduit ${ }^{25}$ dans d'autres philosophies sociales ou politiques, ou qui peut simplement avoir une efficacité sociale très marginale voire quasiment nulle. La comptabilité est donc, pour le sociologue, une entrée intéressante dans l'analyse de phénomènes et processus plus larges. 


\section{Notes}

1. On a donné plusieurs noms à ce courant et domaine de recherche, on a parlé de "sociologie des sciences ", de "sociologie de l'innovation ", de "sociologie des réseaux socio-techniques".

2. Le premier exemple s'insère dans une recherche beaucoup plus large portant sur les réformes économiques chinoises et plus particulièrement les réformes concernant les entreprises d'État. Nous avons passé pour cette recherche dix huit mois en Chine répartis en six séjours de 1993 à 2001, et réalisé une centaine d'entretiens en langue chinoise auprès de directeurs, directeurs financiers et directeurs du personnel d'entreprises d'État, de personnes travaillant dans des services bancaires en relation avec ces entreprises, de chercheurs et enseignants-chercheurs au sein d'instituts de sociologie, économie et comptabilité, et enfin de comptables et officiels en charge des questions comptables. Nous avons également participé à deux colloques internationaux organisés par le ministère des finances chinois, l'un en 1993 en tant qu'observatrice, le second en 1994 en tant qu'interprète (une délégation française était présente) ; le premier était destiné à faire le point sur la réforme comptable de 1993, le second à en étudier les "nécessaires " développements.

Le deuxième exemple s'appuie, premièrement sur des entretiens réalisés auprès des personnes chargées des finances dans notre université, deuxièmement sur notre participation à différents conseils, commissions, sous-commissions, etc., ces différentes expériences ayant toutes été appréhendées comme des enquêtes de terrain appliquant une méthode d'observation particulière qu'est l'observation participante, troisièmement sur un certain nombre de rapports réalisés sur ces questions, que, pour la plupart, nous n'avons pas fait figurer en bibliographie.

3. Par exemple, l'analyse des débats ayant engagé les représentants des professions de santé, y compris les psychologues, lors de la mise au point de la nouvelle nomenclature (1982), éclaire les modes concurrents de construction d'une identité professionnelle (par le diplôme, l'expérience...) et per- mettent de comprendre les compromis inscrits dans la nomenclature : pp. 46-51.

4. Pour une présentation du courant ethnométhodologique, on peut se reporter à Coulon (1987) ; pour une réflexion ethnométhodologique sur les catégories à Raisons pratiques (1994).

5. Les économistes néo-institutionnalistes ont également réfléchi à cette question, mettant l'accent, quant à eux, sur l'existence de " configurations institutionnelles "; l'école de la régulation a ainsi montré la complémentarité des arrangements ou compromis institutionnels qui contribuent à la viabilité d'un mode de régulation ; de nombreux travaux de l'école des conventions ont également insisté sur la cohérence et la continuité de formes conventionnelles très diverses, par exemple dans un même modèle de production industrielle tel le fordisme (Thévenot, 1985).

6. Nos sources, outre les entretiens réalisés, sont essentiellement de langue chinoise ; les informations en langue plus accessible sont rares, on peut se référer en français à Gervais et Wang (1992), en anglais à Tang (1994). Pour des informations plus détaillées que le cadre général et partiel, car orienté par nos objectifs, que nous présentons ici, le mieux est de se reporter à Gervais et Wang, et à Eyraud (2003).

7. Jusqu'au milieu des années 1980, la comptabilité n'existe pas en tant que discipline indépendante, elle est simplement considérée comme une spécialité des finances publiques.

8. On peut également le traduire par «Tableau d'équilibre des capitaux " comme le font Gervais et Wang (1992).

9. Ceci dit, cette conception, même si elle se base sur l'économie politique marxiste, est également congruente avec un système économique dans lequel l'État, outre son rôle de percepteur d'impôt, assume ceux de propriétaire et de banquier.

10. Là aussi nous avons travaillé essentiellement sur des sources en langue chinoise, mais une explicitation plus précise de cette réforme peut se trouver dans Tang, 1994, et en langue française dans Évraert et Ding (2000), ou Morin et al. (1996).

11. Nous restons là volontairement à un niveau très général, au niveau « fondateur » en quelque sorte ; 
Corine EYRAUD

COMPTABILITÉ (PUBLIQUE ET D'ENTREPRISE) ET SOCIOLOGIE, OU L'ANALYSE SOCIOLOGIQUE DES CATÉGORISATIONS SOCIALES

on peut ensuite élaborer une typologie des différentes conceptions ayant existé dans le monde capitaliste.

12. D'autres réflexions récentes proposent de reconnaître le capital humain en tant qu'actif de l'entreprise, et par exemple la formation comme un investissement immatériel (voir Capron, dans Colasse, 2000, pp. 271-278 ; et Lecointre, dans Colasse, 2000, en particulier pp. 981-982). Cela dit, de manière générale " la reconnaissance d'un actif ou d'un passif a toujours été au cœur de la problématique comptable» (Simon, dans Colasse, 2000, p. 1247).

13. Voir Capron (2003).

14. On trouve une présentation de ces différentes expériences ainsi qu'une bibliographie, entre autres, dans Panoramiques (2000), pp. 94-168.

15. Voir Colette et Richard (2002), pp. 10-29.

16. Nous avons également par ailleurs (Eyraud, 2003, pp. 503-507) analysé cette efficacité à travers les décisions bancaires d'octroi ou refus de prêts, et développé une analyse plus approfondie de l'efficacité de la réforme de manière générale.

17. Le directeur d'une agence bancaire française installée en Chine nous signalait d'ailleurs que les directeurs d'entreprises chinoises venaient presque toujours le trouver en lui demandant d'investir dans leur entreprise, et lui s'évertuait à leur expliquer qu'il n'investissait pas mais qu'il prêtait de l'argent. Ceci dit, la difficulté de différencier les subventions et les investissements de l'État ne semble pas particulière aux entreprises d'État chinoises. Le PDG d'une entreprise publique française interrogé pour une émission radiophonique de France Inter ("Rue des entrepreneurs") en octobre 1996, déclarait qu' " il est toujours difficile de faire la différence entre une subvention et une augmentation de capital ».

18. Cette manière de poser les problèmes ainsi que les conclusions qui en découlent ne sont possibles que si les salariés n'apparaissent dans les comptes de l'entreprise qu'à travers leurs rémunérations considérées comme des charges et non pas en tant qu'actif de l'entreprise (cf la note $\mathrm{n}^{\circ} 12$ ). Lecointre disait, à propos de ce choix de comptabilisation des salariés, qu' " il est à craindre que (ce choix) soit celui de comptes non seulement aveugles mais potentiellement mortiferes. Ces pratiques comptables pourraient être mises en examen et avoir quelques comptes à rendre au procès des licenciements s'il s'ouvrait un jour. " (dans Colasse, 2000, p. 982).

19. Le compte de résultat met au jour l'importance de leurs pertes, le bilan leur haut niveau d'endettement, l'importance de la dette inter-entreprises et des créances douteuses, voire irrécouvrables.

20. La réduction de personnel ou la gestion des personnels d'entreprises mises en faillite se fait à travers l'invention d'une nouvelle catégorie de sanstravail : les xiagang (littéralement ceux qui sont descendus de poste). Ces estimations proviennent de Rocca (2000), l'auteur y explique les incertitudes de ces statistiques (d'où une fourchette aussi importante) et les modalités de gestion sociale de cette catégorie. Le taux de réemploi de cette catégorie varie selon les sources de 20 à $70 \%$.

21. Étant donné qu'un des objectifs essentiels de la comptabilité est à cette époque de fournir des informations pour la planification nationale, le lien entre système comptable et système statistique est très fort : ce sont le plus souvent les catégories statistiques qui informent les catégories comptables, et les préoccupations du bureau national des Statistiques qui l'emportent dans l'élaboration de ces systèmes comptables et financiers. Son influence sur la question comptable va aller en s'amenuisant tout au long des années 1980 jusqu'à la réforme comptable de 1993, cf Eyraud (2003).

22. Il est intéressant de noter que l'on semble aller vers une totalisation de l'activité universitaire, alors que depuis le $19^{\text {ème }}$ siècle on connaît un processus de fragmentation de l'unité de l'activité économique (Donzelot, 1984 ; Salais, 1986).

23. Nous n'entrons pas dans les détails, les non-spécialistes peuvent se reporter à Lande, dans Colasse (2000), pp. 193-209. Les spécialistes, quant à eux, sauront de quoi il est question.

24. Les outils et indicateurs pour ce contrôle de gestion restent pour beaucoup à élaborer, mais ce travail a déjà commencé au sein de la Direction interministérielle à la réforme de l'État (Dire) et de son centre de ressources consacré au dévelop- 
Corine EyRAUD

pement du contrôle de gestion dans les administrations. "Un document pratique réalisé par la Dire, largement distribué dans l'ensemble des administrations de l'État contribue à la diffusion de cette nouvelle culture " (Service public, 2002, p. 25).

25. Jusqu'à présent, très peu de voix opposées à la LOLF, voire même nuancées, se sont fait entendre ; cela a commencé mais de manière dispersée au sein du mouvement social du printemps dernier ; ces voix pourraient prendre de l'ampleur en fonction des modes d'application à venir de la LOLF et des incidences que ces modes pourraient avoir.

26. Qui n'est peut-être pas si nouveau que ça d'ailleurs, mais qui pour l'instant a eu, dans la plupart des administrations, peu d'efficacité sociale, et qui, par la LOLF, devrait ou pourrait en avoir plus. Au moins un cas particulier est toutefois à noter : celui des établissements hospitaliers qui ont déjà largement connu ces évolutions (Halgand, dans Colasse, 2000, pp. 357-369). Il semble désormais convenu d'appeler ce nouvel esprit le "nouveau management public " (Hood, 1995).

27. Nous avons fait le choix de travailler essentiellement la dimension catégorisation sociale de la comptabilité normalisée ; face à d'autres choix, d'autres approches sociologiques pourraient être mobilisées : l'ethnométhodologie, par exemple, si l'on souhaite travailler sur la comptabilité pratiquée, mais aussi la théorie des champs de Bourdieu et/ou la sociologie des professions et de l'expertise si l'on souhaite travailler sur le champ ou la profession comptable (Ramirez, 2003), et la sociologie des sciences si l'on analyse des controverses comptables (voir par exemple Miller, 1991, ou Robson, 1993).

28. Nous empruntons ce concept à la sociologie de la traduction (Callon, 1986).

\section{Bibliographie}

Amblard M. (2002), Comptabilité et conventions, L'Harmattan.

BOLTANSKI L. (1982), Les cadres - la formation d'un groupe social, Editions de Minuit.
Boltanski L. et Chiapello E. (1999), Le nouvel esprit du capitalisme, Gallimard.

Bourdieu P. (1982), Ce que parler veut dire, Fayard.

Bourdieu P. (1993), "A propos de la famille comme catégorie réalisée ", Actes de la recherche en sciences sociales, $\mathrm{n}^{\circ} 100$, pp. 32-36.

Callon M. (1981), « Pour une sociologie des controverses technologiques ", Fundamenta Scientiae, vol. 2, n 3/4, pp. 381-399.

Callon M. (1986), "Elements pour une sociologie de la traduction ", L'Année sociologique, $\mathrm{n}^{\circ} 36$, pp. 169-208.

CALlon M. (éd.) (1989), La science et ses réseaux. Genèse et circulation des faits scientifiques, La Découverte.

Capron M., Leseul G. (1997), « Pour un bilan sociétal des entreprises ", Revue des études coopératives mutualistes et associatives (RECMA), $\mathrm{n}^{\circ} 266, \mathrm{pp}$. 28-41.

Capron M. (2003), "Jean-Baptiste Godin : entrepreneur de son siècle ou bâtisseur du futur ?", communication aux Neuvièmes journées d'histoire de la comptabilité et du management.

CENTRE DES JEUNES DIRIGEANTS D'ENTREPRISE (CJD) (1996), L'entreprise au XXIe siècle, Flammarion.

Colasse B. (2003), Comptabilité Générale, Economica.

COlasse B. (dir.) (2000), Encyclopédie de comptabilité, contrôle de gestion et audit, Economica.

Colette C. et Richard J. (1996), Comptabilité générale. Une optique internationale, Dunod.

COLETTE C. et RiCHARD J. (2002), Les systèmes comptables français et anglo-saxons, Dunod.

CONFÉRENCE DES PRÉSIDENTS D'UNIVERSITÉ (2001), Autonomie des Universités, Actes de colloque.

CONFÉRENCE DES PRÉSIDENTS D'UNIVERSITÉ (2002), Budget global des Universités, Rapport du groupe de travail Mudry.

Coulon A. (1987), L'ethnométhodologie, PUF, Que sais-je ?

Desrosières A. et Thévenot L. (1988), Les catégories socioprofessionnelles, La Découverte.

Desrosières A. (1993), La politique des grands nombres - Histoire de la raison statistique, La Découverte. 
DesRosières A. (2000), « L'usage des statistiques dans l'étude des inégalités sociales ", dans Définir les inégalités, des principes de justice à leur représentation sociale, DREES/MIRE.

DONZELOT J. (1984), L'Invention du social, essai sur le déclin des passions politiques, Fayard.

DuRKHeIM E. (1912), Les formes élémentaires de la vie religieuse, Alcan.

ÉvraerT S. et Ding Y. (2000), "Comptabilité en Chine ", dans Colasse (dir.), pp. 291-300.

Eyraud C. (1999), L'entreprise d'État chinoise : de "l'institution sociale totale" vers l'entité économique ?, L'Harmattan.

Eyraud C. (2003), « Pour une approche sociologique de la comptabilité - Réflexions à partir de la réforme comptable chinoise ", Sociologie du Travail, $\mathrm{n}^{\circ}$ 4, pp. 491-508.

FOREST F. (2003), «L'« entreprise État » et les discours sur la réforme des lois de finances ", Quaderni, n 53 , pp. 13-21.

Gervais M. et Wang H.T. (1992), "Les principes de la comptabilité chinoise ", Revue française de Comptabilité, no 240, pp. 89-103.

Guillaume H., Dureau G. et Silvent F. (2002), Gestion publique. L'État et la performance, Presses de Sciences Po et Dalloz.

Hood C. (1995), "The "New Public Management" in the 1980s : Variations on a Theme ", Accounting, Organizations and Society, vol. 20, n $2 / 3$, pp. 93-109.

LATOUR B. (1984), Les microbes. Guerre et paix, A.M. Métailié.

LATOUR B. (1989), La science en actions, La Découverte.

Miller P. (1991), « Accounting Innovation Beyond the Enterprise : Problematizing Investment
Decisions and Programming Economic Growth in the UK in the 1960s ", Accounting, Organizations and Society, vol. 16, $\mathrm{n}^{\circ} 8$, pp. 733762.

Miller P. (2000), « How and Why sociology Forgot Accounting ", Papier présenté à la Conférence du $25^{\mathrm{e}}$ anniversaire d'Accounting, Organizations and Society.

Morin A., Pairault T. et Wu C. (1996), Le plan comptable des entreprises industrielles chinoises, EHESS et Ordre des experts-comptables.

Musselin C. (2001), La longue marche des universités françaises, PUF.

PANORAmiques (2000), La démocratie dans l'entreprise : une utopie ?, $\mathrm{n}^{\circ} 46$.

QUÉRÉ L. (1994), "Présentation ", Raisons pratiques, $\mathrm{n}^{\circ} 5$, pp. 7-40.

RAISONS PRATIQUES (1994), L'enquête sur les catégories - De Durkheim à Sacks, $n^{\circ} 5$.

RAMireZ C. (2003), « Du commissaire aux comptes à l'audit : les Big4 et la profession comptable française depuis 1970 ", Actes de la recherche en sciences sociales, $\mathrm{n}^{\circ} 146-147$, pp. 62-79.

Robson K. (1993), "Accounting Policy Making and Interests ", Critical Perspectives on Accounting, vol. 4, $\mathrm{n}^{\circ} 1$, pp. 1-27.

RocCA J.-L. (2000), « La montée du chômage dans la Chine urbaine ", Perspectives chinoises, $\mathrm{n}^{\circ}$ 59, pp. 38-51.

Salais R., Baverez N. et Reynaud B. (1986), Linvention du chômage, PUF.

SERVICE PUBLIC (2002), Le contrôle de gestion, un outil de pilotage, $\mathrm{n}^{\circ} 91$.

TANG (dir.) (1994), Accounting and Finance in China, a Review of Current Practice, Longman Group. 
3498_02_Eyraud 22/04/2004 11:22 Page 46

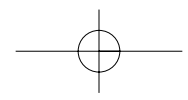

